\title{
Wound complication among different skin closure techniques in the emergency cesarean section: a randomized control trial
}

\author{
Bhimeswar Nayak G, MBBS, Pradip Kumar Saha, MBBS, MD, MAMS, FIMSA, Rashmi Bagga, MBBS, MD, \\ Bharti Joshi, MBBS, MD, Minakshi Rohilla, MBBS, MD, Shalini Gainder, MBBS, MD, Pooja Sikka, MBBS, MD \\ Department of Obstetrics and Gynecology, Post Graduate Institute of Medical Education and Research, Chandigarh, India
}

\section{Objective}

Cesarean section is the most commonly performed obstetrical surgical procedure; however, there are no standard guidelines on appropriate skin closure techniques and materials. Only few comparative studies have been conducted on different skin closure techniques, and they have shown conflicting results. Therefore, we compared different skin closure techniques during emergency cesarean section to identify the best technique with minimal wound complication rates.

\begin{abstract}
Methods
Patients were randomized into 3 groups (group $A, n=100$; group $B, n=102$; and group $C, n=98$ ). In group $A$, the skin was closed using staples; in group B, via the subcuticular technique using monocryl 3-0; and in group C, using mattress suture nylon (2-0). The primary outcome was a composite of wound complications, including infection, seroma, gaping, and need for resuturing and antibiotic administration. The secondary outcome included closure time, pain perception, patient satisfaction, and cost. Analyses were performed in accordance with the intention-to-treat principle.
\end{abstract}

Results

The composite wound complication rate in the entire cohort was $16.6 \%(n=50)$; the complication rate was significantly higher in group A than in the other groups. Infection was the most common wound complication observed in the entire study group ( $86 \%)$ and was significantly higher in group $A$ than in groups $B$ and $C(P \leq 0.001)$.

\section{Conclusion}

The use of staples for cesarean section skin closure is associated with an increased risk of wound complications and prolonged hospital stay postoperative visits.

Keyword: Caesarean section; Wound complication; Skin closure techniques

\section{Introduction}

Cesarean section (CS) is the most common obstetrical procedure performed worldwide, including India. Despite its high prevalence, data regarding the preferred surgical techniques, including those for skin closure, are limited. Skin closure is an integral step of CS; it influences cosmesis and patient and surgeon satisfaction and is associated with the absence or presence of wound complications [1,2]. As the national and international CS rates are increasing continuously, the number of wound complications, such as seroma, hema-
Received: 2018.12.17. Revised: 2019.07.02. Accepted: 2019.07.15. Corresponding author: Pradip Kumar Saha, MBBS, MD, MAMS, FIMSA

Department of Obstetrics and Gynecology, Post Graduate Institute of Medical Education and Research, Sector 12, Chandigarh 160012, India

E-mail: pradiplekha@yahoo.co.in

https://orcid.org/0000-0002-3200-4124

Articles published in Obstet Gynecol Sci are open-access, distributed under the terms of the Creative Commons Attribution Non-Commercial License (http://creativecommons. org/licenses/by-nc/3.0/) which permits unrestricted non-commercial use, distribution, and reproduction in any medium, provided the original work is properly cited.

Copyright $\odot 2020$ Korean Society of Obstetrics and Gynecology 


\title{
Obstetrics \& Gynecology Science
}

\author{
Vol. 63, No. 1, 2020
}

toma, infection, separation, and dehiscence, in CSs may also be expected to increase. In developing countries, such as India, the exact rate of wound complications following CS is not known, and there are limited data regarding the effect of specific skin closure techniques on wound healing at the time of CS. Underlying medical maternal disorders, immunosuppression, steroid intake, obesity, frequent vaginal examinations, emergency $C S$, and failure to close or drain the subcutaneous tissue more than $2 \mathrm{~cm}$ in thickness are the common risk factors for CS wound complications. The ideal skin closure technique should be safe, effective, and inexpensive, take less time, and yield minimal patient discomfort and good cosmetic outcomes. It should also require minimal follow-up evaluation and yield a low rate of wound complications $[3,4]$.

In our center, the commonly used techniques for skin closure include the use of interrupted non-absorbable sutures (monofilament nylon, 2-0), subcuticular sutures (monocryl $2-0$ ), and staples. Mattress sutures have the advantages of being inexpensive, easily available, and comfortable for use among surgeons and of having good tissue-holding strength at a deeper level and less risks of wound dehiscence. However, they have the disadvantages of being associated with increased risks of infection (higher with silk than with monofilament nylon) and poor cosmesis and of having the need for stitch removal [5-8]. Staples are preferred because of their rapid applicability, easy compatibility, and good cosmetic outcomes but are costly and may be associated with a higher rate of wound dehiscence $[8,9]$. Subcuticular sutures using monocryls yield good cosmetic outcomes; however, their use has the disadvantage of being time-consuming and costly $[8,9]$.

Only a few randomized controlled trials have compared different skin closure techniques after abdominal surgeries and their wound complications; however, none of them have concluded which technique is the best for Pfannenstiel incision closure following CS. A Cochrane review in 2012 also found no conclusive evidence on the best technique for skin closure after CS [9]. Hence, we compared different skin closure techniques during emergency CS to identify the technique associated with minimal wound complication rates.

\section{Materials and methods}

This study was a randomized controlled trial conducted in the Department of Obstetrics and Gynecology, Postgraduate Institute of Medical Education and Research, Chandigarh over a period of 14 months (January 2013 to February 2014). The study was approved by our Institutional Ethics Committee (reference number: NK/755/MD/1686-1687). Written consent was obtained from all patients at recruitment. In this trial, there were 1,514 women who were screened, and a total of 307 patients fulfilled the inclusion criteria and were then enrolled in the study after randomization. Seven women dropped out of the study at the time of CS. Women aged 18-40 years undergoing primary emergency CS via Pfannenstiel incision and at gestation of more than 28 weeks with a maternal weight of less than $75 \mathrm{~kg}$ were enrolled in the study. Women with previous uterine scars or hypersensitivity to suture materials; active lesions on the anterior abdominal wall; and history of other medical conditions, such as uncontrolled diabetes mellitus, abnormal renal function test results, coagulopathy, clinical icterus, human immunodeficiency virus/hepatitis B surface antigen/hepatitis C virus infection, anemia (hemoglobin level of $<7 \mathrm{gm} \%$ ), shock, fever due to any cause, clinical chorioamnionitis, autoimmune disorders, immunosuppression, and drug addiction were excluded from the study; those with chronic steroid use and who were smokers were also excluded.

Detailed obstetrical and medical histories were noted. The patients underwent thorough examinations, including anthropometric evaluation (height and weight), and their information was recorded in detail.

The patients' baseline hemoglobin level was assessed, and other antenatal investigations were performed as per the existing protocol and according to the individual case. The types of onset of labor (induced/spontaneous), duration of term/preterm rupture of membranes (if present), number of vaginal examination, duration of labor, and indication for the emergency CS were noted. Preoperative medications, such as ampicillin (2 g), ranitidine (150 $\mathrm{mg}$ ), and metoclopramide (10 mg; bolus) were administered via intravenous (IV) injection 30 minutes before surgery after checking the antibiotic test dose as per the hospital protocol. An alternative antibiotic, i.e., cefazolin $2 \mathrm{~g}$, was injected intravenously in the patients sensitive to ampicillin.

A total of 300 women were randomized using the tippet random number table method into 3 groups (group $A$, $n=100$; group $B, n=102$; and group $C, n=98$ ) after the decision to perform CS. All surgeries were performed by the 


\section{Obstetrics \& Gynecology Science}

Bhimeswar Nayak G, et al. Skin closure in cesarean section

consultant or senior resident. The surgical procedure was the same for all 3 groups until closure of the rectal sheath, which was conducted using No. 1 polydioxanone.

In group $A$, the subcutaneous fat was closed using plain catgut (2-0) and interrupted sutures and the skin using staples (material: stainless steel; Covidien, Dublin, Ireland).

In group $B$, the subcutaneous fat was closed using interrupted sutures of monocryl (polyglecaprone 25, 3-0; Ethicon, Somerville, NJ, USA) and $3 / 8$ circle cutting needles; the skin was closed via the subcuticular technique using the same suture material.

In group C, subcutaneous fat was closed using plain catgut $(2-0)$ when it was $>2 \mathrm{~cm}$ in thickness, followed by skin closure using mattress sutures of monofilament nylon (2-0) with a reverse cutting needle.

A pressure bandage was applied after spirit cleansing in all patients.

\section{Wound complications}

Herein, hematoma was defined as a mass of usually clotted blood that forms in a tissue organ or body space as a result of a broken blood vessel. Seroma was classified as a mass or tumefaction caused by localized accumulation of serum within a tissue or organ. Wound separation was defined as any separation of the wound that was identified as such by the patient or via medical record review and varied in size from small skin defects to separation of the entire wound [10].

\section{Intraoperative details}

The thickness of the subcutaneous fat at the midline of the skin incision and time taken for surgery, subcutaneous fat closure, and skin closure were noted. The estimated blood loss amount, number of units of blood transfused (if any), and any intraoperative complications (e.g., postpartum hemorrhage and bladder or bowel injury) were noted in each patient. Immediate postoperative IV fluids (oxytocin drip), analgesics (diclofenac), and antibiotics (ampicillin $500 \mathrm{mg} \mathrm{IV}$ 6th hourly or cefazolin $1 \mathrm{~g}$ IV 8th hourly for the patients sensitive to ampicillin) were administered on postoperative day 0 . Immediate postoperative problems, such as fever, soakage of dressing, and any anaphylactic reaction, were noted. The IV cannula and Foley catheter were removed on postoperative day 1. IV antibiotic was switched to oral amoxicillin $500 \mathrm{mg}$ 8th hourly (capsule) and analgesic to diclofenac $50 \mathrm{mg}$ 8th hourly (tablet), which were administered up to postopera- tive day 5. At postoperative day 2, the aseptic dressing was opened, and the suture line status was assessed. During this time, any discharge from the stitch line (details regarding type and amount, if present), any induration present, hematoma, and any other signs of inflammation were recorded. As a part of the routine hospital protocol, hemogram, routine urinary examination, and urinary culture were performed. If any discharge from the stitch line was present, a swab was taken in a sterile condition and sent for microbiological culture and sensitivity analysis. If required, additional antibiotics were added as per the treating clinician's decision. If added for induration, any serratiopeptidase derivatives were noted, and any systemic signs of infection, such as fever, tachycardia, and myalgia, were recorded. If indicated, additional investigations, including workup for fever, were performed on an individual basis. Any fever, discharge from the wound, wound gaping, and induration present from postoperative day 2 to day 7 were noted and managed accordingly. The patients were discharged as per the treating clinician's decision any time after postoperative day 2 . The duration of postoperative hospital stay was recorded.

All patients who were discharged earlier than postoperative day 7 were advised to visit for follow-up on postoperative day 7. Staple removal was conducted in group A, suture removal in group $C$, and stitch line assessment in all groups. Pain on the stitch line was assessed using a 10-point pain scale in which patients were asked to rate their pain and their responses were then noted [11]. Patient satisfaction was measured using a Likert scale [12] (poor, fair, good, and very good) based on the technique of skin closure, its cost, wound outcome, suture/staple removal, pain perception, and whether patients would desire to receive the same skin closure technique for future delivery. Thereafter, patients were followed up via telephone interviews up to 6 weeks. Decisions regarding additional antibiotics, frequency of dressing, readmission, and secondary suturing (if required) were made as per the treating clinician's decision. The total cost associated with the technique used and management of its associated wound complications were assessed in each patient by summing up individual costs for the material used for incision closure, additional antibiotics used, and additional hospital stay required owing to wound concerns. Other costs, such as those for the dressing material and staffing, were not included. Data on readmission, if performed owing to wound concerns, were noted, including the postoperative 


\section{Obstetrics \& Gynecology Science}

Vol. 63, No. 1, 2020

day of readmission, need for additional antibiotics, frequency of dressing needed, and details of resuturing, if required.

The primary outcomes measured were the number of patients who had seroma, hematoma, skin separation, and wound infection and those who needed additional antibiotics and resuturing. The secondary outcomes measured were the skin closure time, pain perception on postoperative day 7 , patient satisfaction (Likert scale) [12], and cost analysis.

\section{Statistical analysis}

Statistical analysis was conducted using the SPSS software, version 15.0 for Windows (SPSS Inc., Chicago, IL, USA). Means and medians were calculated for quantitative variables and standard deviations or standard errors for mea- sures of dispersion. For skewed data, the Mann-Whitney test was applied. Proportions were compared using the $\chi^{2}$ test or Fisher's exact test, as applicable. One-way analysis of variance was applied to compare the means of quantitative data among the 3 groups with Bonferroni correction for the post hoc multiple comparison test. All statistical tests were 2-sided and performed at a significance level of $\alpha=0.05$ ( $P$-value). For Bonferroni correction for multiple comparisons among the 3 groups, the significance level was adjusted to $P<0.017$.

\section{Results}

The baseline and clinical characteristics of the study population are described in Table 1. All 3 groups were comparable

Table 1. Baseline and clinical characteristics of 3 study groups

\begin{tabular}{|c|c|c|c|c|}
\hline Characteristics & Group A $(n=100)$ & Group B $(n=102)$ & Group C $(n=98)$ & $P$-value \\
\hline \multicolumn{5}{|l|}{ Baseline characteristics } \\
\hline Age & $27.0 \pm 4.3$ & $26.5 \pm 3.8$ & $26.5 \pm 4.1$ & 0.16 \\
\hline Primigravida (\%) & 59 & 61 & 45 & 0.04 \\
\hline Period of gestation & $36.3 \pm 2.7$ & $36.2 \pm 2.5$ & $36.6 \pm 2.5$ & 0.96 \\
\hline Maternal weight (kg) & $60.2 \pm 6.6$ & $60.7 \pm 6.3$ & $60 \pm 6.2$ & 0.63 \\
\hline \multicolumn{5}{|l|}{ Clinical characteristics } \\
\hline Hemoglobin (gm/dL) & $10.8 \pm 1.5$ & $11.1 \pm 1.5$ & $11 \pm 1.6$ & 0.31 \\
\hline Induction of labour (\%) & 43 & 41 & 47 & 0.63 \\
\hline PN examination & $2.57 \pm 1.6$ & $2.04 \pm 1.3$ & $2.19 \pm 1.1$ & 0.06 \\
\hline DROM (hr) & $9.6 \pm 16.3$ & $7.3 \pm 7.1$ & $14.9 \pm 22$ & 0.07 \\
\hline Duration of labour (hr) & $6.57 \pm 5.3$ & $5.72 \pm 5.2$ & $5.64 \pm 4.3$ & 0.41 \\
\hline GDM/DM (\%) & 4 & 7 & 7 & 0.58 \\
\hline
\end{tabular}

Data are given as mean \pm standard deviation not otherwise specified.

PN, pervaginal; DROM, duration of rupture of membrane; GDM, gestational diabetes; DM, diabetes mellitus.

Table 2. Surgical characteristics

\begin{tabular}{|c|c|c|c|c|}
\hline Surgical characteristics & Group A $(n=100)$ & Group B $(n=102)$ & Group C ( $n=98)$ & $P$-value \\
\hline Subcutaneous fat depth (cm) & $2.75 \pm 0.30$ & $2.68 \pm 0.30$ & $2.69 \pm 0.40$ & 0.61 \\
\hline Subcutaneous fat closure (min) & $1.61 \pm 0.50$ & $1.60 \pm 0.40$ & $1.58 \pm 0.50$ & 0.71 \\
\hline Duration of surgery (min) & $55.9 \pm 9.0$ & $52.0 \pm 9.7$ & $54.5 \pm 11.5$ & 0.69 \\
\hline \multicolumn{5}{|l|}{ Indication for cesarean } \\
\hline Fetal distress & 69 & 67 & 72 & \\
\hline Meconium & 10 & 13 & 09 & \\
\hline Malpresentation & 14 & 13 & 11 & \\
\hline Others & 07 & 09 & 06 & \\
\hline
\end{tabular}

Data are given as mean \pm standard deviation not otherwise specified. 


\section{Obstetrics \& Gynecology Science}

Bhimeswar Nayak G, et al. Skin closure in cesarean section

in terms of age, booking status, maternal weight, and period of gestation. There were $59 \%, 61 \%$, and $45 \%$ primigravidas in groups $A, B$, and $C$, respectively; however, the difference was not significant. There was also no significant difference among the 3 groups in terms of the mean hemoglobin level, induction of labor, duration of rupture of membranes, number of vaginal examination prior to lower segment CS, duration of labor, and number of women with diabetes mellitus $(P \geq 0.05)$.

Surgical characteristics, including subcutaneous fat depth, subcutaneous fat closure time, and surgical duration (Table 2), were assessed and were found to be comparable among the 3 groups.

To compare the primary outcomes among the 3 study groups, the Pearson $\chi^{2}$ test was applied (Table 3 ). The overall composite wound complication rate for the entire cohort was $16.6 \%(n=50)$; the complication rate was significantly higher in group $A$ than in the other groups. Out of the total of 50 women with wound complications, $60 \%$ were from group $A$; this was highly significant when group $A$ was compared with group $B$ and group $C(P=0.002$ and $P<0.001$, re- spectively). Infection was the most common wound complication observed in the entire study group, and the infection rate was significantly higher in group $A$ than in groups $B$ and $C(P \leq 0.001)$. Nine of the 27 women and 2 of the 8 women with wound infection in groups $A$ and $B$ developed wound gaping, respectively. In group C, 8 had wound infection, and 3 had wound gaping. Resuturing was required in 4 women with wound gaping; the remaining cases were allowed to heal by secondary intention.

In group A, 1 woman developed 5-cm wound gaping just above the rectal sheath, which was managed with daily dressing and additional antibiotics. The wound swab culture was sterile in this patient. Three women developed complete wound dehiscence just above the rectal sheath along with high-grade fever, which required wound debridement and administration of antipyretics and additional antibiotics. The wound swab culture of 1 of these 3 women showed growth of Acinetobacter, which was sensitive to amikacin. However, the blood cultures of all 3 women were sterile. All of them required secondary resuturing on postoperative day 14 , and 2 of them required readmission. The cost associated with

Table 3. Primary outcomes in 3 different groups

\begin{tabular}{|c|c|c|c|c|}
\hline Primary outcomes & Group A $(n=100)$ & Group B $(n=102)$ & Group C ( $n=98)$ & $P$-value \\
\hline Wound complications & 30 & 12 & 8 & $<0.001^{b)}$ \\
\hline Seroma & 3 & 4 & 0 & 0.78 \\
\hline Haematoma & 0 & 0 & 0 & 0.99 \\
\hline Wound gaping ${ }^{a)}$ & 9 & 2 & 3 & 0.11 \\
\hline Wound infection & 27 & 8 & 8 & $<0.001^{\text {b) }}$ \\
\hline Resuturing & 3 & 1 & 0 & 0.07 \\
\hline Additional antibiotics & 11 & 2 & 4 & 0.01 \\
\hline
\end{tabular}

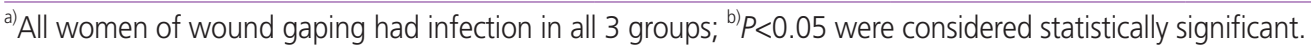

Table 4. Secondary outcomes in different groups

\begin{tabular}{|c|c|c|c|c|}
\hline Secondary outcomes & Group A $(n=100)$ & Group $B(n=102)$ & Group C $(n=98)$ & $P$-value \\
\hline Skin closure time(min) & $0.68 \pm 0.30$ & $5.68 \pm 0.70$ & $4.29 \pm 1.00$ & $<0.001^{\text {a) }}$ \\
\hline \multicolumn{5}{|l|}{ Pain perception (D7) } \\
\hline A) Mild & 76 & 91 & 92 & 0.009 \\
\hline B) Moderate & 18 & 10 & 5 & 0.009 \\
\hline C) Severe & 6 & 1 & 1 & 0.009 \\
\hline Good patient satisfaction & 63 & 76 & 47 & $<0.001$ \\
\hline Total cost (Rs) & $543.20 \pm 410.41$ & $371.10 \pm 120.75$ & $186.20 \pm 144.75$ & $<0.001$ \\
\hline
\end{tabular}

Rs, Rupee (currency of India).

${ }^{\text {a) }} P<0.05$ were considered statistically significant. 


\title{
Obstetrics \& Gynecology Science
}

\author{
Vol. 63, No. 1, 2020
}

wound complication management was 5 times higher than that in the women in the same group with no complications.

In group B, 1 of the 2 women with wound gaping required multiple dressings, administration of additional antibiotics and antipyretics, and secondary resuturing. The cost associated with wound complication management was 4 times higher than that in the patients in the same group with no complications. In group C, 3 women developed wound gaping, which was managed conservatively and healed by secondary intention. The number of patients who needed additional antibiotics was significantly higher in group $A$ than in the other groups $(P=0.01)$. When associated factors, i.e., hemoglobin level, onset of labor, duration of rupture of membranes, number of vaginal examination, indication and duration of surgery, and amount of blood loss, were compared between the women with and without wound complications, no significant difference was found.

The secondary outcomes are presented in Table 4. Although the skin closure time was the shortest with closure using staples, the women who received such reported significantly higher pain levels at postoperative day 7 . The mean total cost for the skin closure technique and its associated wound complications was much higher in group A than in groups B and C.

\section{Discussion}

The best skin closure technique and material to use and the outcome associated with each remain unclear. Initial small studies comparing various skin closure techniques reported contradictory results. Previous studies mainly focused on the potential impact of CS skin closure techniques on pain or cosmesis $[13,14]$. Recently, some studies, including clinical trials and meta-analyses, have compared various techniques of skin closure and addressed wound morbidities [5,15-17].

The composite wound complication rate calculated in our study was $16.6 \%$, which is in line with that reported by Basha et al. [5] Generally, we observed that surgical staples were associated with a significantly higher incidence of composite wound morbidity than were subcuticular and mattress sutures. The higher rate of wound infection in group $A$ may be attributed to the allergic or inflammatory reactions owing to the metallic nature of staples and greater wound disruptions. Our observation is in agreement with that of few studies that the rate of wound separation is higher with the use of staples $[5,16]$. The higher rate of wound separation was attributed to early staple removal in few studies $[5,14]$. However, in our study, staple removal was performed on postoperative day 7. Most of the wound separation cases were minor and superficial and needed only few additional dressings without readmission.

Herein, we considered seroma, hematoma, wound gaping, and wound infection as wound complications. There is a wide range of wound complications cited in the literature; this is attributed to the various definitions of wound infection and several associated factors among various studies $[15,18,19]$. Conversely, lower wound complication rates have been reported in studies with broad exclusion criteria than in studies with stringent exclusion criteria, and there is no uniformity regarding antibiotic prophylaxis. In our study, we continuously administered prophylactic antibiotics to all women until postoperative day 7 . Since the completion of this study, we started to administer only prophylactic antibiotics within 1 hour before the surgery. A high complication rate was found in our study despite the broad exclusion criteria and antibiotic coverage; this could be attributed to the fact that our institute is a tertiary care center with majority of cases referred from other hospitals, and all patients included underwent emergency CSs unlike in other studies [5,13,14,20-22]. Further, $2.3 \%$ of the women in our study had wound seroma; this was not measured as an outcome in other studies. We think our data on the different wound complications are accurate, as wound assessment was performed objectively, unlike in other studies where it was conducted subjectively [5]. No woman in our study group developed wound hematoma; however, this could be because of the small sample size. Thus, it is difficult to form any conclusions on this rare outcome. The higher wound complication rate observed in the patients who received closure using staples than in those who received such via the subcuticular technique (30\% vs. $12 \%$ ) is in accord with that of a previous systematic review and meta-analysis, where it was concluded that subcuticular sutures are associated with lower risks of wound complications in CS [16].

Various studies have shown staples to be superior in terms of the closure time; however, the findings related to postoperative pain, cosmesis, and cost-effectiveness are not consistent $[5,14,23]$. Indexed studies reported shorter skin closure time and higher pain levels on postoperative day 7 , which 


\section{Obstetrics \& Gynecology Science}

Bhimeswar Nayak G, et al. Skin closure in cesarean section

are in line with most reports $[5,19,20]$.

Only a few studies have compared the cosmetic outcomes of different types of sutures. Yang et al. [24] concluded that intradermal vertical mattress sutures are superior cosmetically to subcuticular sutures. Greater patient and physician satisfaction and better cosmetic outcomes were observed with the suture closure method than with the staple closure method [25].

The strength of our study was that it was a randomized controlled trial conducted in a tertiary care teaching institute. We could not compare the wound infection rates in groups with different body mass indices; however, the mean weights of all 3 groups were similar. We observed a 4 -fold greater financial cost incurred when staples were used for skin closure. Basha et al. [5] had similar observations that sutures were 4 times cheaper than staples.

We also observed that skin incisions closed using staples were more likely to have wound complications than those closed using monocryl subcuticular sutures and nylon mattress sutures when placed according to our aforementioned protocol. The plausibility of this association may be attributed to the increased bacterial colonization in the gaps between staples or to the less tissue remodeling and healing with staples as a result of decreased inflammatory response. Another possible explanation may be the better approximation and increased tensile strength with sutures.

We conclude that the use of staples for skin closure for CS is associated with an increased risk of wound complications and postoperative visits. Despite patients' preferences for subcuticular sutures, our data suggest no difference in patient satisfaction after wound healing between subcuticular and mattress sutures. As the present study was a pilot study, the small sample size can be considered our limitation. Further studies with larger sample sizes are needed to delineate our findings.

\section{Conflict of interest}

No potential conflict of interest relevant to this article was reported.

\section{Ethical approval}

Study was approved by Institute Ethics Committee of PGIMER, Chandigarh (Approval No. NK/755/MD/1686-1687).

\section{Patient consent}

Written consent was obtained from all patients while recruiting for study and sharing data.

\section{References}

1. Del Valle GO, Combs P, Qualls C, Curet LR. Does closure of camper fascia reduce the incidence of postcesarean superficial wound disruption? Obstet Gynecol 1992;80:1013-6.

2. Naumann RW, Hauth JC, Owen J, Hodgkins PM, Lincoln T. Subcutaneous tissue approximation in relation to wound disruption after cesarean delivery in obese women. Obstet Gynecol 1995;85:412-6.

3. Jenkins TR. It's time to challenge surgical dogma with evidence-based data. Am J Obstet Gynecol 2003;189:4237.

4. Pearl ML, Rayburn WF. Choosing abdominal incision and closure techniques: a review. J Reprod Med 2004;49:662-70.

5. Basha SL, Rochon ML, Quiñones JN, Coassolo KM, Rust OA, Smulian JC. Randomized controlled trial of wound complication rates of subcuticular suture vs staples for skin closure at cesarean delivery. Am J Obstet Gynecol 2010;203:285.e1-8.

6. Zuber TJ. Soft tissue surgery for the family physician. In: Skin biopsy, excision, and repair techniques: an illustrated guide for the family physician, book three. Kansas City (MO): American Academy of Family Physicians; 1998. p.100-6.

7. Stasko T. Advanced suturing techniques and layered closures. In: Wheeland RG, editor. Cutaneous surgery. Philadelphia (PA): Saunders; 1994. p.304-17.

8. Moy RL, Lee A, Zalka A. Commonly used suturing techniques in skin surgery. Am Fam Physician 1991;44:162534.

9. Mackeen AD, Berghella $V$, Larsen ML. Techniques and 


\section{Obstetrics \& Gynecology Science}

Vol. 63, No. 1, 2020

materials for skin closure in Caesarean section. Cochrane Database Syst Rev 2012;11:CD003577.

10. Kawakita T, Landy HJ. Surgical site infections after cesarean delivery: epidemiology, prevention and treatment. Matern Health Neonatol Perinatol 2017;3:12.

11. Jensen MP, Tomé-Pires $C$, Solé $E$, Racine $M$, Castarlenas $E$, de la Vega $R$, et al. Assessment of pain intensity in clinical trials: individual ratings vs composite scores. Pain Med 2015;16:141-8.

12. Voutilainen A, Pitkäaho T, Kvist T, Vehviläinen-Julkunen K. How to ask about patient satisfaction? The visual analogue scale is less vulnerable to confounding factors and ceiling effect than a symmetric Likert scale. J Adv Nurs 2016;72:946-57.

13. Frishman GN, Schwartz T, Hogan JW. Closure of Pfannenstiel skin incisions. Staples vs. subcuticular suture. J Reprod Med 1997;42:627-30.

14. Rousseau JA, Girard K, Turcot-Lemay L, Thomas N. A randomized study comparing skin closure in cesarean sections: staples vs subcuticular sutures. Am J Obstet Gynecol 2009;200:265.e1-4.

15. Clay FS, Walsh CA, Walsh SR. Staples vs subcuticular sutures for skin closure at cesarean delivery: a metaanalysis of randomized controlled trials. Am J Obstet Gynecol 2011;204:378-83.

16. Tuuli MG, Rampersad RM, Carbone JF, Stamilio D, Macones GA, Odibo AO. Staples compared with subcuticular suture for skin closure after cesarean delivery: a systematic review and meta-analysis. Obstet Gynecol 2011;117:682-90.

17. Mackeen AD, Schuster M, Berghella V. Suture versus staples for skin closure after cesarean: a metaanalysis. Am J Obstet Gynecol 2015;212:621.e1-10.
18. Siddiqui DS, Lacuna EM, Chen HY, Chauhan SP. Skin closure of pfannenstiel incision with dermabond, staples, or suture during cesarean delivery: experience of a single attending. Am J Perinatol 2013;30:219-24.

19. Islam A, Ehsan A. Comparison of suture material and technique of closure of subcutaneous fat and skin in caesarean section. N Am J Med Sci 2011;3:85-8.

20. Koigi-Kamau R, Kabare LW, Wanyoike-Gichuhi J. Incidence of wound infection after caesarean delivery in a district hospital in central Kenya. East Afr Med J 2005;82:357-61.

21. Burke JJ 2nd, Gallup DG. Incisions for gynecologic surgery. In: Rock JA, Jones HW 3rd, Te Linde RW, editors. Te Linde's operative gynecology.10th ed. Philadelphia (PA): Wolters Kluwer/Lippincott Williams \& Wilkins; 2008. p.246.

22. Figueroa D, Jauk VC, Szychowski JM, Garner R, Biggio JR, Andrews WW, et al. Surgical staples compared with subcuticular suture for skin closure after cesarean delivery: a randomized controlled trial. Obstet Gynecol 2013;121:33-8.

23. Cromi A, Ghezzi F, Gottardi A, Cherubino M, Uccella S, Valdatta L. Cosmetic outcomes of various skin closure methods following cesarean delivery: a randomized trial. Am J Obstet Gynecol 2010;203:36.e1-8.

24. Yang J, Kim KH, Song YJ, Kim SC, Sung N, Kim H, et al. Cosmetic outcomes of cesarean section scar; subcuticular suture versus intradermal buried suture. Obstet Gynecol Sci 2018;61:79-87.

25. Fleisher J, Khalifeh A, Pettker C, Berghella V, Dabbish N, Mackeen AD. Patient satisfaction and cosmetic outcome in a randomized study of cesarean skin closure. J Matern Fetal Neonatal Med 2019;32:3830-5. 